Steinisch, Martin; Sulpizio, Valentina; lorio, Angelo Andrea; Di Naccio, Alessandra; Haueisen, Jens; Committeri, Giorgia; Comani, Silvia:

A virtual environment for egocentric and allocentric mental transformations: a study on a nonclinical population of adults with distinct levels of schizotypy

Zuerst erschienen in: Biomedizinische Technik = Biomedical Engineering. - Berlin [u.a.] : de Gruyter. - 56 (2011), 5, p. 291-299.

Erstveröffentlichung: $\quad$ 2011-10-12

ISSN (online): $\quad$ 1862-278X

ISSN (print): 0013-5585

DOI: $\quad$ 10.1515/BMT.2011.107

[Zuletzt gesehen: 43692]

„Im Rahmen der hochschulweiten Open-Access-Strategie für die Zweitveröffentlichung identifiziert durch die Universitätsbibliothek IImenau."

"Within the academic Open Access Strategy identified for deposition by Ilmenau University Library."

„Dieser Beitrag ist mit Zustimmung des Rechteinhabers aufgrund einer (DFGgeförderten) Allianz- bzw. Nationallizenz frei zugänglich."

"This publication is with permission of the rights owner freely accessible due to an Alliance licence and a national licence (funded by the DFG, German

DFG

Research Foundation) respectively." 


\section{A virtual environment for egocentric and allocentric mental transformations: a study on a nonclinical population of adults with distinct levels of schizotypy}

\author{
Martin Steinisch ${ }^{1, *}$, Valentina Sulpizio ${ }^{2,3}$, Angelo \\ Andrea Iorio ${ }^{4}$, Alessandra Di Naccio ${ }^{4}$, Jens \\ Haueisen $^{5}$, Giorgia Committeri ${ }^{1-3}$ and Silvia \\ Comani $^{1,2,6}$ \\ ${ }^{1}$ BIND - Behavioral Imaging and Neural Dynamics \\ Center, University "G. d'Annunzio", Chieti, Italy \\ 2 Department of Neuroscience and Imaging, University "G. \\ d'Annunzio", Chieti, Italy \\ ${ }^{3}$ ITAB - Institute for Advanced Biomedical Technologies, \\ "G. d'Annunzio" Foundation, Chieti, Italy \\ ${ }^{4}$ Faculty of Psychology, University "G. d'Annunzio", \\ Chieti, Italy \\ ${ }^{5}$ BMTI - Institute of Biomedical Engineering and \\ Informatics, Ilmenau University of Technology, Ilmenau, \\ Germany \\ ${ }^{6}$ Casa di Cura Privata Villa Serena, Città S. Angelo, Italy
}

\begin{abstract}
We benefited from the flexibility provided by virtual reality to enhance a classical paradigm on array and self mental rotations and related questions on a set of items. We used this paradigm to investigate how the Level of Schizotypy in nonclinical subjects might influence their behavior in egocentric and allocentric mental transformations. Three elements of novelty were introduced: (i) we separated the phases of mental transformation (Imagined Rotation Phase) and task performance (Task Phase), (ii) we measured the time required for Imagined Rotation Phase and Task Phase separately, and (iii) we cued self-rotations with a virtual human being (selfavatar) or an inanimate object (self-chair). Twenty-four nonclinical participants were categorized in low- and highschizotypal subjects (Low-S, High-S). A mixed-design analysis of variance showed that High-S were significantly faster than Low-S during the Imagined Rotation Phase (array and self-chair rotations) and during the Task Phase (self-chair). High-S were also faster in the self-chair than in the selfavatar rotation, supporting the existence of a dissociation between perspective changing and perspective taking in High-S. In line with the literature, we found that participant performances decreased with increasing angular difference between the initial and the imagined perspective.
\end{abstract}

\footnotetext{
*Corresponding author: Martin Steinisch, BIND - Behavioral Imaging and Neural Dynamics Center, University

"G. d'Annunzio", Via dei Vestini 33, 66100 Chieti, Italy

Phone: + 39-0871-3556901

Fax: +39-0871-3556930

E-mail: m.steinisch@unich.it
}

Keywords: mental rotations; perspective taking; schizotypal disorder; virtual reality.

\section{Introduction}

The ability of taking another person's point of view is essential in everyday life and provides crucial information for social interactions. Visual perspective transformations have been extensively explored by using tasks in which participants are asked to look at an array of objects on a turntable and to imagine themselves rotating around the array (for a review see [30]). The goodness of the underlying spatial updating process has been mainly tested through two classes of questions: appearance questions ask subjects to judge how the whole array appears from the new perspective, whereas item questions ask about the location of array items relative to the new perspective (e.g., [19]).

Whereas item questions are likely to cue a geometric strategy, appearance questions are suggested to require a simulation of the first-person experience associated with a different perspective [16]. According to Langdon and colleagues, this characteristic would be critical for the effects they recently observed in nonclinical subjects with a high degree of schizotypy and in patients with a diagnosis of schizophrenia [16, 17]. Langdon and colleagues indeed observed a selective difficulty in answering to appearance questions, but not to item questions, after an imagined selfrotation. It is worth noting that the same subjects did not show any deficit in performing both tasks after an imagined rotation of the whole array $[16,17]$. In this case, subjects would use their own body as a stable egocentric frame of reference to which they would refer the rotation of objects, whereas during mental self-rotations subjects would use the array (and/or the surrounding environment) as a fixed allocentric frame of reference.

According to the authors, individuals with a high degree of schizotypy, or affected by schizophrenia, show an imbalance between egocentric (internal or self-centered reference) and allocentric (external or world-centered reference) mental transformations, with a disadvantage for the latter when associated with an appearance task. Poor mentalizing in schizophrenia would be better understood as a perspective taking impairment, rather than a disruption of a cognitive module dedicated to computing meta-representations [17].

Unfortunately, the relative contribution of abilities underpinning the Imagined Rotation Phase and the Task Phase is impossible to appreciate in the reported studies, because of the physical experimental setup, and also because of the 
single instruction given to the subjects, which regarded both the rotation (self vs. array) and the task to perform after the rotation (item vs. appearance).

In this study, we aimed at complementing previous findings by disentangling the mental processes engaged in each of the two phases of perspective taking assessment, and by comparing self-rotations primed by different cues, such as a virtual chair (as in the studies by Langdon and colleagues) and an avatar, used in previous studies to investigate the ability to take the point of view of others in space $[2,11$, $13,24,26]$. To that purpose, instead of using physical devices, we implemented our paradigm taking advantage of the flexibility provided by a virtual reality (VR) environment, therefore improving the versatility of the paradigm by increasing the number of variables that can be manipulated and measured. In our paradigm, directly inspired to the work by Langdon et al. [16, 17], subjects with low and high schizotypy were studied while performing mental or visuospatial transformations centered on egocentric and allocentric frames of reference, as assessed with array-rotations and self-rotations (primed by a chair or by an avatar), each followed by classical appearance and item questions. Although egocentric and allocentric are terms generally used in the literature to describe self-rotations and array-rotations, respectively (see [30] for a review), here we use these terms in accordance with Langdon and colleagues, thus differentiating them on the basis of which frame of reference remains fixed during the visuospatial transformation (i.e., the egocentric frame of reference during an array-rotation, and the allocentric frame of reference during a self-rotation).

We recorded both the time needed to imagine the rotations and the time required to perform the following task, as well as the number of correct responses, hence deepening the knowledge of cognitive processes underlying visual perspective taking and their relationship with schizotypal personality traits.

\section{Materials and methods}

\section{Subjects}

Twenty-four nonclinical volunteers were enrolled for this study after written informed consent. The study was performed in compliance with the World Medical Association Declaration of Helsinki regarding ethical conduct of research involving human subjects. Volunteers were 12 females (average age $27.3 \pm 5.4$ years) and 12 males (average age $28.4 \pm 4.0$ years). All subjects were evaluated for their schizotypy level, as assessed by the Schizotypal Personality Questionnaire (SPQ) [20], a self-report scale of 74 yes/no items comprising nine subscales (ideas of reference, excessive social anxiety, odd beliefs or magical thinking, unusual perceptual experiences, odd or eccentric behavior, no close friends, odd speech, constricted affect, suspiciousness), which were designed to give a dimensional assessment of the nine schizotypal personality disorder features, as defined in the "diagnostic and statistical manual of mental disorders, $3^{\text {rd }}$ ed., revised" (DSM-III-R) [1]. For our study, we used the Italian translation of SPQ published by Fossati et al. [7].
The median of the total SPQ score (11.5) was used to split the study sample into two groups: one of low-schizotypal (Low-S) subjects and one of high-schizotypal (High-S) subjects. Each group included 12 subjects (Low-S group: 5 females and 7 males, average age $28.8 \pm 5.4$ years; High-S group: 7 females and 5 males, average age $27.5 \pm 4.5$ years). The mean SPQ rating of Low-S subjects was $5.5(\mathrm{SD}=3.5)$, compared to $18.2(\mathrm{SD}=6.6)$ for the High-S subjects. All our subjects, from both Low-S and High-S sub-groups, obtained SPQ scores well below 41, suggested in the original publication of the Schizotypal Personality Questionnaire [20] as a high cut-off value for a probable clinical diagnosis of schizotypal personality disorder.

\section{Virtual environment}

To avoid providing the participants any ambient reference that might affect the results of our study, the virtual environment consisted of an artless room and a circular, one-legged table. The complete layout was rotationally symmetric: the room itself had a quadratic footprint, the table was placed exactly in the center of the room, all walls were without decorations (including doors or windows) and both floor and ceiling had rotationally symmetric textures. All virtual objects were modeled either with 3ds Max (http://www. autodesk.com/3dsmax) or Blender (http://www.blender.org), two modeling and rendering software packages. To create the large number of diverse experimental trials necessary for our paradigm, the actual placement and visualization of the objects, as well the registration of the subjects' responses (both times and correctness), were handled by XVR (http:// www.vrmedia.it/Xvr.htm), a framework for creating and visualizing high-quality $3 \mathrm{D}$ content.

Furthermore, the subjects were not allowed to freely navigate in the virtual room. Instead they were shown several perspectives of the table, which differed from each other by angular steps of $90^{\circ}$. A total of four angles of rotation was used: $0^{\circ}$, corresponding to the subject's perspective in the initial phase of mental rotation, and $90^{\circ}, 180^{\circ}$ and $270^{\circ}$ (arranged counterclockwise around the table), which were randomly used in the Task Phase. Because of the symmetry of the room, the participants could distinguish the different perspectives only by focusing on the arrangement of four colored balls (yellow, green, red and blue), symmetrically placed on the table.

Because the whole experimental design was based on the use of static images showing a low number of nonmoving objects, we estimated that an immersive VR system would not be necessary to comprehend the scene and respond adequately. Instead, an off-the-shelf $19^{\prime \prime}$ monitor was placed at a distance of $40 \mathrm{~cm}$ in front of the subjects and used for visualization. To avoid potential distracting visual stimuli, the experiment was performed in a darkened room.

\section{Imagined Rotation Phase}

During the Imagined Rotation Phase participants were asked to imagine to perform a rotation so that the set of balls would 
result rotated by a given angle. Rotations might be imagined in two different ways: either by rotating the set of balls or by taking a different perspective of the observed scene. Here, we describe the details of the two possible imagined rotations.

Array-rotations In the case of array-rotations, subjects were requested to imagine rotating the set of balls placed on the table, while keeping their position fixed with regard to the table. They thus had to use an egocentric frame of reference in this case. Unlike Langdon and Coltheart [16], we did not use a turntable in our VR environment, because we wanted to keep the visualization as simple as possible, and also because we wanted to avoid potential confusion on how the turntable would work mechanically. After testing several graphical realizations, we concluded that using a virtual pan would be a more plausible and less confusing visualization (Figure 1A, left side). Because the colored balls were placed inside the pan, it seemed logical that they would rotate accordingly, as the subject imagines to rotate the pan by pulling its handle. Therefore, the instruction for this type of mental rotation reads as follows: "Imagine to pull the handle of the pan directly in front of you and to look at the balls that were rotated accordingly'.

Self-rotations Self-rotations required that the subjects imagined moving themselves relatively to the fixed array of balls placed on the table in the virtual room. Participants thus had to use an allocentric frame of reference in this case. To discriminate between taking someone else's perspective and changing their own perspective, we used, respectively, an avatar or a chair to indicate the position where the subjects should mentally move to. If the end position for the rotation was cued by an avatar, the instruction was: "Imagine to move to the avatar's place and to look at the balls from his perspective". By contrast, if a chair was shown, the instruction was: "Imagine to move and sit in the chair and to look at the balls from that perspective". Examples of scenes representing both types of self-rotation are shown in Figure 1A, center and right side.

Participants were informed that, to solve the following tasks correctly, they had to perform the imagined rotation and memorize the configuration of the four balls after the rotation. No preference was indicated with regard to the direction of imagined rotation (clockwise or counterclockwise). In addition, we performed some control trials in which neither an array nor a self-rotation had to be done. In those trials, we displayed the table with the four balls, but without pan, avatar or chair. Because no indicator for a rotation was given, the subjects only had to memorize the configuration of the balls.

\section{Task Phase}

After the Imagined Rotation Phase participants were asked to perform a task, which could be of two different types, described in detail below.

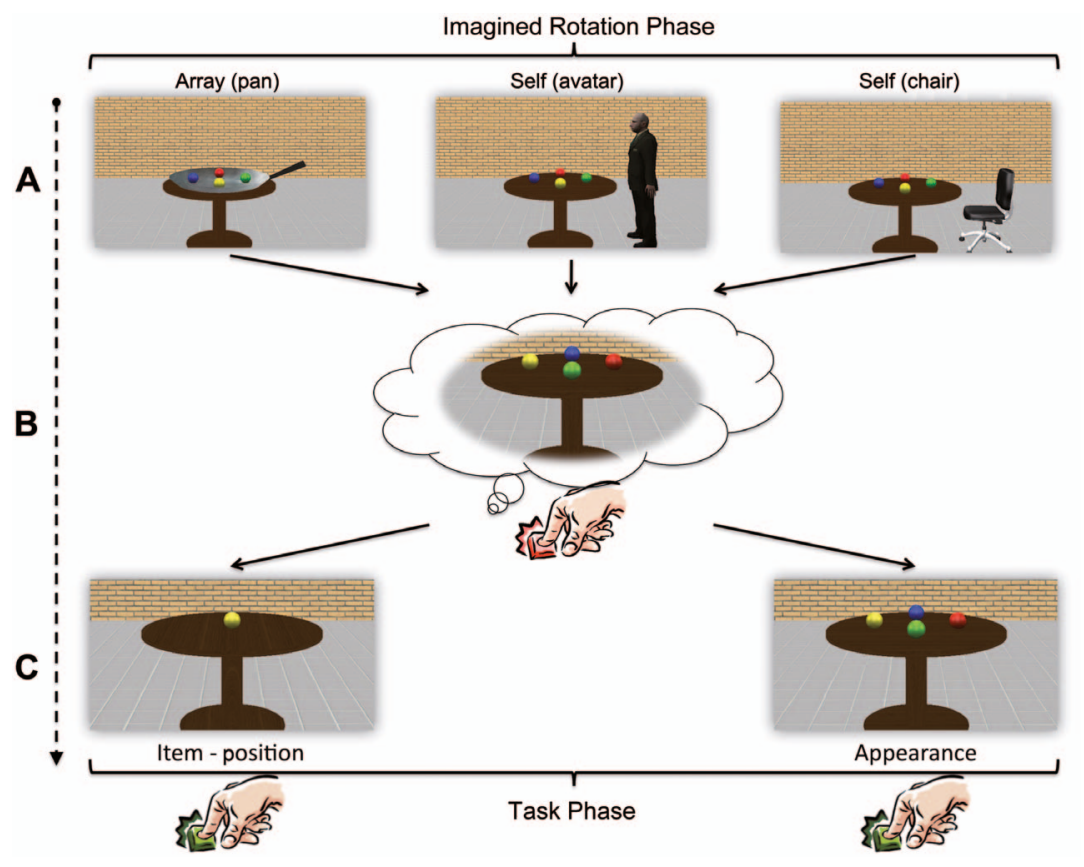

Figure 1 Schematic representation of the experimental design: (A) Imagined Rotation Phase: depending on the presented picture, subjects had to perform either an array-rotation (if the pan was shown) or a self-rotation, if the avatar or the chair were shown. (B) The configuration of the colored balls, after the mental rotation, had to be memorized. A response button was used to measure the time required for this process (Imagined Rotation Time). (C) Task Phase: after each mental rotation, subjects had to respond to either an Appearance Task or an Item-position Task (randomly mixed) by pressing an appropriate response button, hence allowing the measurement of the Task Time and, consequently, of Task Accuracy. 
Appearance Task In this type of task, participants were asked to respond to a question about the appearance of the set of balls from the resulting perspective after the Imagined Rotation Phase. Participants were shown a different perspective of the virtual environment with the four colored balls (Figure 1C, right side), but the configuration of the balls was not necessarily the correct one, as compared to the configuration in the previously performed Imagined Rotation Phase. When the four colored balls were presented, participants knew from instructions provided during the training phase that the question to respond was: "Would the balls look like this after the rotation that you performed?'. For the cases where the correct answer was " $\mathrm{No}$ ", we randomly selected one of the 23 incorrect permutations of the positions of the balls, but we balanced the trials so that in $50 \%$ of all Appearance Tasks the correct answer was "Yes", and "No" in the remaining $50 \%$.

Item-position Task In contrast to the Appearance Task, the virtual scene displayed for an Item-position Task showed only a single colored ball at the center of the table and participants were asked to judge the position of the single ball with regard to the memorized configuration of the balls from the previous Imagined Rotation Phase (Figure 1C, left side). When a single ball was presented, the participants knew from instructions provided during the training phase that the question to respond was: "Is the shown ball on the left or on the right after the rotation that you performed?", hence requiring to answer "Left" or "Right". Similar to Appearance Tasks, both answers had an equal likelihood of 50\%. Constellations where the correct answer would have been "Front" or "Back" were excluded by design to maintain a balance between the number of available answers for both the Appearance and the Item-positions Tasks.

Both Appearance and Item-position Tasks follow a mechanism of mental rotation and utilize the recall of a visual scene, but only the Appearance Task involves a complete matching between the scene displayed during the Task Phase and the one previously imagined during the mental rotation.

\section{Experimental procedures}

In the Imagined Rotation Phase the initial perspective (from $0^{\circ}$ position) was visible for a maximum of $6 \mathrm{~s}$. However, participants were instructed to perform the mental rotation as fast as possible and to press a button when they were finished. This allowed us to measure the time necessary to perform the required mental rotation (called Imagined Rotation Time). If no button was pressed within $6 \mathrm{~s}$, we continued with the Task Phase anyhow. The desired type of mental rotation was indicated solely by displaying an avatar, a chair or a pan in the virtual environment, as described in the previous section. No written or acoustic instructions were used at this stage, and subjects could freely choose the direction of imagined rotation. To avoid potential learning effects, the positions of the colored balls were randomly permuted in each trial.

In the Task Phase, subjects were shown a second image and asked to perform either an Appearance Task or an Item- position Task. As in the Imagined Rotation Phase, no written or acoustic instructions were used, as the task could be clearly determined from the number of objects on the table (4 balls for an Appearance Task and 1 ball for an Item-position Task). Answers were given using predetermined keys of the computer keyboard, corresponding to "Yes" or " $\mathrm{No}$ ", answers in the Appearance Task, and to "Left" or "Right" answers in the Item-position Task. This allowed us to measure the time necessary to perform the task (called Task Time), as well as the response accuracy (named Task Accuracy). As in the Imagined Rotation Phase, we limited the maximum response time to $6 \mathrm{~s}$. If no response was given within this time, the trial was considered as missing and we continued with the next trial.

For both the Imagined Rotation Phase and the Task Phase, the maximum response time (6s) was determined on the basis of preliminary observations on a set of separate subjects naive to the scope of the experiment.

For each type of imagined rotation and for each combination of angular step and task we performed 8 trials, thus resulting in 48 trials ( 3 angles $\times 2$ tasks $\times 8$ trials) for arrayrotations (pan), 48 trials for self-rotations with the avatar and 48 trials for self-rotations with the chair. Because the design of the control condition excluded rotations, only the different tasks could be considered, hence summing up to 16 additional control trials ( 2 tasks $\times 8$ trials). In total, each participant performed 160 trials. To avoid an increase in response times due to tiredness, the 144 experiment trials were grouped in 6 blocks of 24 trials with short breaks in between. Each block contained only a single type of rotation, with Appearance and Item-position Tasks randomly mixed within each block. Therefore, there were 2 blocks for each type of rotation and a single block containing the 16 control trials. To avoid potential undesired learning effects which might result from performing the trials in a specific sequence, the order of blocks was permuted in between subjects.

Before performing the experiment, participants had to successfully complete a training phase to become acquainted with the virtual environment and the experimental tasks, as usual practice in cognitive neuroscience studies. The training trials were similarly designed to the experimental trials described above, and the training session was arranged in 4 blocks, one for each type of imagined rotation (array-rotation with pan, self-rotations with avatar and chair) and one for the control trials (without rotation). Each imagined rotation training block contained 12 trials ( 3 angles $\times 2$ tasks $\times 2$ possible answers), whereas the control training block, due to the lack of different angles, contained only 4 trials ( 2 tasks $\times 2$ possible answers). Participants performed each training block at least once and eventually had to repeat it if they responded correctly to $<75 \%$ of the trials in this particular block.

After the experiment, all subjects were administered a structured interview in which they had to report whether they experienced any difficulties (related to technical issues or to the experimental paradigm itself). They were asked about the actual strategy they had used to perform the tasks. In addition, participants had to compile questionnaires to assess their Level of Schizotypy (Schizotypal Personality Question- 
naire [20]) and their handedness (Edinburgh Handedness Inventory [18]).

\section{Statistical analysis}

Because the experimental paradigm was designed to disentangle the mental processes engaged in both phases of perspective taking assessment (i.e., the Imagined Rotation Phase and the Task Phase), we recorded and analyzed the reaction times during the Imagined Rotation (array-rotation with the pan, self-rotations with the avatar and the chair) and Task Phases, which we will refer to as Imagined Rotation Time and Task Time, respectively, from now onward. We additionally measured subjects' accuracy in performing the tasks (Task Accuracy).

Trials in which the subjects did not respond in time (maximum $6 \mathrm{~s}$ ) or did not answer correctly were excluded. Also, for each subject and each group of directly comparable trials (those with the same imagined rotation, angular step and task), outliers in the Imagined Rotation Time and Task Time distributions were defined as not lying within 2 standard deviations of the mean (mean $\pm 2 * \mathrm{SD}$ ) and were excluded from further analysis.

We compared the performances of the two groups (High$\mathrm{S}$ and Low-S subjects) in a mixed-design analysis of variance (ANOVA) with Rotation (array-pan, self-avatar and selfchair), Angle $\left(90^{\circ}, 180^{\circ}\right.$ and $270^{\circ}$ ) and Task (Appearance and Item-position) as within-subjects factors. In addition, we re-ran the same analyses with the subjects' gender, instead of Level of Schizotypy, as the between-groups factor to check for gender-based differences in Imagined Rotation Time, Task Time or Task Accuracy. Furthermore, we checked for a potential interaction between Gender and Level of Schizotypy.

\section{Results}

\section{Debriefing}

No participant reported any difficulty in understanding the experiment or problems related to technical issues, such as clear visibility of the screen, good differentiation of colors and easiness of input device use. All subjects reported to have used a nonholistic, verbal strategy during the experiment, which means that they performed the required imagined rotation and, afterwards, always used the same order of positions of the balls (e.g., "left, front, right, back') to memorize the corresponding colors with regard to the new, imagined perspective, regardless of the angular step. Most subjects, but not all, realized that it was sufficient to memorize just 3 out of the 4 balls, because we used the same 4 colors during the entire experiment. Hence, if the correct colors for 3 positions are already known, only one possible color is left for the last vacant position in the configuration of the balls.

Based on the administered Schizotypal Personality Questionnaires, the Level of Schizotypy was evaluated for each participant and used to categorize all participants into groups of low- and high-schizotypal subjects (Low-S and High-S), as already described above. All participants were right-handed, as assessed by the Edinburgh Handedness Inventory (mean score $73.10 \pm 19.97$ ).

\section{Imagined Rotation Time}

We analyzed the Imagined Rotation Time (mean value) as a $2 \times 3 \times 3$ factorial design, with Level of Schizotypy as the between-groups factor and Rotation and Angle as repeatedmeasures. The mixed ANOVA on Imagined Rotation Times revealed a significant main effect of Angle $\left(\mathrm{F}_{2,44}=4.07\right.$; $\mathrm{p}=0.024$ ), as well as the two-way interactions between Level of Schizotypy and Rotation $\left(\mathrm{F}_{2,44}=3.37 ; \mathrm{p}=0.044\right)$ and between Rotation and Angle $\left(\mathrm{F}_{4,88}=4.44 ; \mathrm{p}=0.003\right)$. The variable Task was not considered for this analysis because, during the Imagined Rotation Phase, subjects were unaware of the task they would have performed in the Task Phase.

The interaction between Level of Schizotypy and Rotation was due to the fact that High-S subjects spent significantly less time, as compared to Low-S subjects, in the array $(p=0.033)$ and self-chair $(p=0.0002)$ rotations (Figure 2$)$. In the Low-S group, we found no significant differences in the performance of the three types of rotation $(\mathrm{p}>0.05)$, whereas the High-S group performed the self-chair rotation significantly faster than the self-avatar $(\mathrm{p}=0.028)$ and array $(\mathrm{p}=0.044)$ rotations

Additionally, we found behavioral effects that were not affected by the Level of Schizotypy. In particular, the effect of Angle was observed only for the array-rotation (Rotation by Angle interaction, Figure 3), with an increased Imagined Rotation Time when the imagined rotation was of $180^{\circ}$ as compared to both $90^{\circ}(p=0.004)$ and $270^{\circ}(p=0.0003)$. Moreover, no significant difference between rotations of $90^{\circ}$ and $270^{\circ}(\mathrm{p}>0.05)$ was found, indicating that clockwise and counterclockwise mental rotations were equivalent. By contrast, no angular effect was found for the self-centered rotations (avatar and chair), thus reflecting the existence of a chronometric increase of Imagined Rotation Time only for the mental array-rotation.

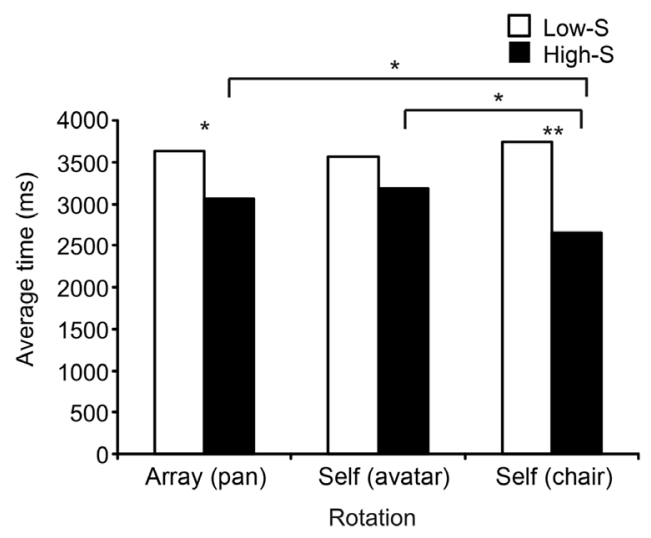

Figure 2 Average Imagined Rotation Times for Low-S and High$\mathrm{S}$ subjects as a function of type of Rotation $(* \mathrm{p}<0.05$; ** $\mathrm{p}<0.001)$. 


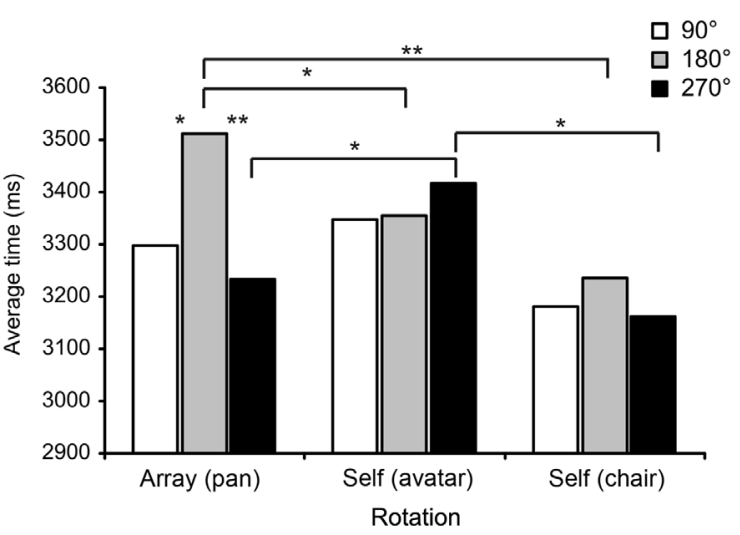

Figure 3 Average Imagined Rotation Times for each rotation as a function of Angle $(* \mathrm{p}<0.05 ; * * \mathrm{p}<0.001)$.

\section{Task Time}

Task Time was analyzed as a function of Level of Schizotypy (between-groups factor), Task, Rotation and Angle (withingroup factors) in a mixed ANOVA. The analysis on reaction times (mean values of correct responses) revealed the main effects of Task $\left(\mathrm{F}_{1,22}=83.86 ; \mathrm{p}=0.00001\right)$ and Angle $\left(F_{2,44}=11.06 ; p=0.0001\right)$, as well as the Level of Schizotypy by Rotation interaction $\left(\mathrm{F}_{2,44}=3.45 ; \mathrm{p}=0.041\right)$. Also the Task by Rotation $\left(\mathrm{F}_{2,44}=3.77 ; \mathrm{p}=0.031\right)$ and the Task by Angle $\left(\mathrm{F}_{2,44}=9.35 ; \mathrm{p}=0.0004\right)$ interactions were significant.

As for the Imagined Rotation Time, we found that High$\mathrm{S}$ subjects were generally faster than Low-S subjects (Figure 4). Consistently with the results on Imagined Rotation Time, the two groups mainly differed in chair rotation $(p=0.0003)$, as High-S subjects spent significantly less time to perform the task after a self-rotation cued by the chair. The two groups did not significantly differ for the other imagined rotations (array and self-avatar). No differences were observed in High-S subjects among all rotations $(p>0.05)$, whereas we observed that Low-S subjects spent significantly more time in performing the task after the self-chair rotation as compared to the array-rotation $(p=0.047)$ and partially with regard to the self-avatar rotation $(\mathrm{p}=0.060)$, indicating

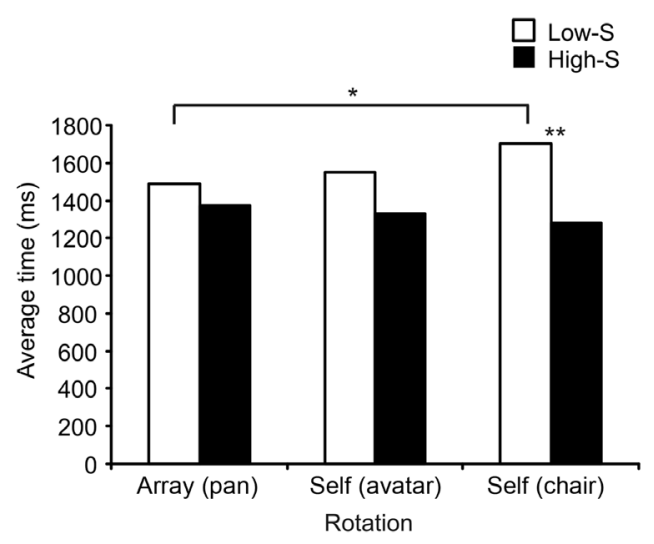

Figure 4 Average Task Times for Low-S and High-S subjects as a function of Rotation $(* \mathrm{p}<0.05 ; * * \mathrm{p}<0.001)$. an opposite trend with regard to the Imagined Rotation Phase.

As shown in Figure 5, we obtained further behavioral effects common to both High-S and Low-S groups. We observed that all subjects, on average, performed the Itemposition Task faster than the Appearance Task (main effect of Task), and that these tasks significantly differed on the basis of the type of rotation performed (Task by Rotation interaction). Indeed, we found that the type of rotation selectively affected the Item-position Task, as Task Time was significantly lower after a self-rotation cued by the avatar, as compared to Task Time after a self-rotation cued by the chair $(p=0.015)$. Conversely, the type of previous rotation did not significantly affect the Appearance Task Time $(\mathrm{p}>0.05)$.

Finally, we found that the increase of reaction time as a function of Angle $\left(90^{\circ}<180^{\circ}>270^{\circ}\right.$ : main effect of Angle) was completely explained by the selective angular effect on the Item-position Task (Task by Angle interaction, Table 1). Here, indeed, we observed that subjects needed more time when the angle of the imagined rotation was $180^{\circ}$ as compared to both $90^{\circ}(\mathrm{p}=0.0013)$ and $270^{\circ}(\mathrm{p}=0.0014)$. Also in this case, we confirmed that clockwise and counterclockwise mental rotations required the same time as no significant difference between $90^{\circ}$ and $270^{\circ}$ was found $(\mathrm{p}>0.05)$.

\section{Task Accuracy}

The mixed ANOVA on subjects' accuracy (number of correct responses), with Level of Schizotypy as between-groups factor, and Task, Rotation and Angle as within-group factors, revealed a main effect of Angle $\left(\mathrm{F}_{2,44}=4.66 ; \mathrm{p}=0.015\right)$, as well as a significant Task by Angle interaction $\left(\mathrm{F}_{2,44}=6.66\right.$; $\mathrm{p}=0.003)$. In this case, no significant interaction with the Level of Schizotypy was found $(\mathrm{p}>0.05)$, thus indicating that High-S and Low-S groups did not significantly differ on the basis of Task Accuracy.

In accordance with the results reported above, we found that the angle of imagined rotation significantly affected subjects' performance only in the Item-position Task. In particular, subjects' accuracy decreased during Item-position Task for imagined rotations of $180^{\circ}$ with regard to $90^{\circ}(\mathrm{p}=0.0029)$

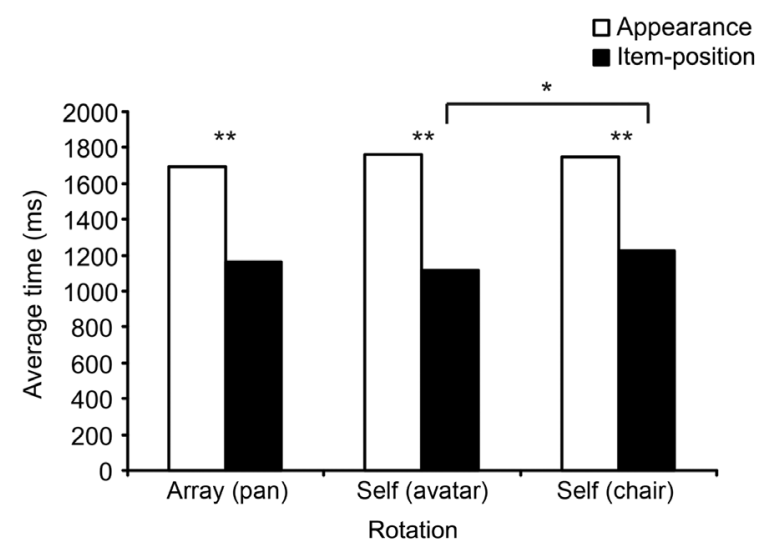

Figure 5 Average Task Times for Appearance and Item-position Tasks as a function of Rotation $(* \mathrm{p}<0.05 ; * * \mathrm{p}<0.001)$. 
Table 1 Averaged times (ms) during Imagined Rotation Phase and Task Phase and averaged accuracy (\% of correct responses) during Task Phase, both with regard to the angular steps.

\begin{tabular}{llll}
\hline & \multirow{2}{*}{$\begin{array}{l}\text { Imagined } \\
\text { Rotation Phase }\end{array}$} & \multicolumn{2}{l}{ Task Phase } \\
\cline { 3 - 4 } & & Appearance & Item-position \\
\hline Time $(\mathrm{ms})$ & & 1754 & 1084 \\
$90^{\circ}$ & 3275 & 1739 & 1328 \\
$180^{\circ}$ & 3368 & 1700 & 1121 \\
$270^{\circ}$ & 3270 & & \\
Accuracy $(\%)^{\mathrm{a}}$ & & 96.01 & 96.70 \\
$90^{\circ}$ & & 94.97 & 91.67 \\
$180^{\circ}$ & & 94.62 & 97.92 \\
$270^{\circ}$ & & & \\
\hline
\end{tabular}

For the Task Phase, measures are subdivided based on the two distinct Tasks, to illustrate that the main effect of Angle was due to the subjects' performance in the Item-position Task.

${ }^{\mathrm{a}}$ Accuracy is not applicable during Imagined Rotation Phase.

and $270^{\circ}(\mathrm{p}=0.0003)$ (Table 1). Also in this case, we did not find any significant differences between performance at $90^{\circ}$ and $270^{\circ}(\mathrm{p}>0.05)$, hence concluding that clockwise and counterclockwise mental rotations were performed in an equivalent manner.

\section{Gender}

In all previous analyses (Imagined Rotation Time, Task Time and Task Accuracy), we additionally checked for potential differences between female and male subjects, by using the gender as between-groups factor (instead of Level of Schizotypy), while leaving the within-group factors as already described above. Based on our sample group, consisting of 12 females and 12 males, no significant main effect of Gender or interactions between Gender and the other factors were observed $(\mathrm{p}>0.05)$. Given the slightly imbalanced distribution of females and males in the two groups of Low-S ( 5 females and 7 males) and High-S (7 females and 5 males), we additionally checked for a potential interaction between gender and Level of Schizotypy. Based on our sample group, no significant interaction was found $(\mathrm{p}>0.05)$. Hence, both gender subgroups provided equal performance throughout the whole experiment.

\section{Discussion}

In the present research, we studied array-rotations and selfrotations by taking advantage of VR, which offers a unique tool to investigate how humans interact with their surroundings under realistic conditions. Indeed, participants can experience a realistic environment with three-dimensional objects and characters, with the advantage that VR allows for more flexibility than a real physical experimental setup, for a precise control over stimuli and for the recording of quantities impossible to measure in real settings, such as the time necessary to imagine a rotation, disentangling it from the time required to perform the task after the mental rotation.
Virtual environments have been previously used for investigating spatial perception and memory relative to different frames of reference [4, 23] (for a recent review, see [9]), as well as first and third person perspective taking [5, 11, 24, 27]. VR setups have been successfully employed in several recent studies on spatial updating [21, 25, 28], and VR appears as a highly promising tool also in the field of mental disease investigation (for a recent review, see [8]).

However, classical paradigms investigating mental arrayrotations and self-rotations, paired with item questions and appearance questions, have been performed only using either real devices (e.g., [19, 29]) or completely imagined setups [3]. Therefore, our study extends the investigations on mental transformations to virtual reality environments.

Inspired by the work of Langdon and Coltheart [16], the present study focused on the relationship between mental transformations and the personality trait of schizotypy measured on a sample of nonclinical young adults. Our results confirm the findings by other authors, as reported further on, but also add some novel interesting outcome, obtained owing to the versatility of VR. To our knowledge, for instance, we are the first to record the time needed to imagine the rotations (self or array) with no task execution, thus disentangling the underlying perspective updating processes from those required to perform the following tasks (appearance or item). Moreover, we compared two types of self-rotations, primed by two different cues: a chair and an avatar. The first should prompt operations of changing own perspective, whereas the second should induce to take the perspective of another individual. It is worth noting that both types of mental rotations require transformations of the egocentric frame [29], but in our paradigm they are conceived as allocentric rotations because they are referred to an external, fixed array of objects [16].

As mentioned several times already, the first main finding of the present study concerns the quantification of the Imagined Rotation Time, and provides new evidence on the relationship between schizotypy and visual perspective taking abilities. We observed that the Level of Schizotypy in nonclinical subjects influences the velocity at which participants perform both array-rotations and self-rotations cued by the chair (same cue as in the experiment by Langdon and Coltheart [16]), with High-S subjects performing faster than Low-S subjects. However, this outcome might not be completely explained as a general tendency of High-S subjects to respond faster because of their impulsiveness. In fact, High-S subjects did not differ from Low-S subjects in the self-avatar condition. Moreover, being faster in the Imagined Rotation Phase did not negatively affect High-S subjects' accuracy during the Task Phase.

The more rapid execution observed in High-S subjects during mental array-rotations has been previously reported by Langdon and Coltheart [16], but selectively for the Appearance Task, thus demonstrating that High-S subjects are good performers when they are asked to judge how the array of items would appear from another perspective after having imagined the array-rotation relative to their own fixed perspective. By separating the Imagined Rotation Phase from 
the Task Phase, we were able to discover that the faster performance of High-S subjects in array-rotations is already present during the mental rotation phase.

With regard to the faster execution observed in High-S subjects during self-rotations cued by the chair, this condition was instead found as impaired when paired with the Appearance Task by Langdon and Coltheart [16], who proposed an interpretation in terms of perspective taking deficit in High$\mathrm{S}$ subjects. However, if perspective taking is the ability to imagine how a visual scene would appear from the viewpoint of another observer [10], and not simply from a different perspective, it should be better studied in the presence of another observer, able to prime a potentially conflicting perspective of the world. In our paradigm, indeed, visual perspective taking has been explored by asking subjects to take the perspective of a virtual human being (avatar). Importantly, this imagined rotation was performed significantly slower by our High-S group (and not by the Low-S group) than the imagined rotation cued by the chair. We claim that this effect is a novel evidence supporting the existence, in nonclinical High-S subjects, of a dissociation between changing their own perspective (self-chair) or taking the perspective of another individual (self-avatar), with an advantage for the former. Various explanations could be provided for this specific result, such as a preference for an inanimate or impersonal cue, or an inhibition by a cue resembling another human being. Both explanations could be associated with the known difficulty of High-S subjects in mentalizing [15].

An indirect confirmation of the insights reached with the analysis of the Imagined Rotation Phase comes from the reaction times recorded during the Task Phase. Indeed, we continue to observe an interaction between the Level of Schizotypy and the type of mental rotation performed before task execution. Once more, a special role was played by the selfchair rotation, because High-S subjects were generally faster than Low-S subjects in executing the tasks after a self-chair rotation. Interestingly, we observed in both groups that the Item-position Task was performed faster after the avatar-cued rotation than after the chair-cued rotation, thus reflecting a dissociation in self-rotations. This advantage for the selfavatar rotation in combination with an Item-position Task can be tentatively explained by the fact that engaging in another's perspective seems to be an automatic process that facilitates the detection of spatial displacements of objects in the scene across perspective changes [2,13]. The Level of Schizotypy, instead, did not appear to influence the performance in the spatial updating tasks, differently from Langdon and Coltheart [16], who described an impairment of High-S subjects during Appearance Tasks after self-rotations. This specific discrepancy between their results and ours might be due to differences between the protocols (Langdon and Coltheart did not differentiate between Imagined Rotation Phase and Task Phase, as we do) or the setups used (real vs. virtual environment and objects), and requires further investigations.

Apart from the effect of schizotypy level, our VR paradigm proved to be sensible to known effects, described in previous experiments. For example, consistent with the results of Langdon and Coltheart [16], we reported that all subjects were faster during Item-position Tasks than during Appearance Tasks, probably because they cue different cognitive strategies by relying on the access to a single element of the imagined array and on the matching between the imagined and the actual mental array configuration, respectively. Furthermore, we found an increase of Imagined Rotation Time as a function of the angular difference between the initial and the imagined perspective, but only for array-rotations [19, 29]. This angular effect followed the classic rotation function established by Shepard and Metzler [22] for mental rotation of objects, with increasing latencies up to $180^{\circ}$ and a drop-off beyond $180^{\circ}$. The lack of an angular effect for self-rotations is contrary to some previous evidence (e.g., [6]) but in accordance with others [19, 29], and might be due to a blink transformation [12], which instantaneously transports the participant to the new perspective irrespective of its distance. These results are, in general, in accordance with all previous studies (reviewed in [30]), indicating faster and more accurate performances following an imagined movement of viewpoint around an array rather than an equivalent imagined rotation of the array.

\section{Conclusions}

In light of the results discussed above, we consider that this study proved the versatility of VR and its potentially high contribution in the development of cognitive sciences. The larger number of variables, that can be manipulated and measured in a virtual environment, lead to an enhancement of a classical paradigm on mental transformations (self or array) and questions (item or appearance) on a set of objects, with a consequential improved understanding of the mental processes engaged in each of the two phases of perspective taking assessment. This allowed us to obtain interesting suggestions on the mechanisms underlying egocentric and allocentric mental transformations in relation to the Level of Schizotypy. Particularly interesting are the findings about the presence of a character (avatar) in the scene, which is only possible in VR environments.

We wish to underline that the described experiment made use of a reasonably moderate VR setup, which nonetheless proved to be sufficient to improve our understanding of the mechanisms of different types of mental transformations. We imagine that an immersive VR system might help to further emphasize the differences we observed among subjects. A further improvement of the virtual environment used in our paradigm might also regard the development of new avatars that could differ by sex and appearance, and that could also embody different types of emotions. We might then, for instance, investigate the influence of emotions on mental rotation performance in subjects with a high Level of Schizotypy.

Another interesting prosecution of the present study could be the use of our VR system and experimental paradigm in a clinical population of schizophrenic patients, who have difficulties in mentalizing and a reduced performance in 
allocentric simulations, like the High-S subjects [17]. More recent results [14] also highlighted that schizophrenic patients have difficulties in basic allocentric spatial perception and in switching between allocentric and egocentric frames of reference. It would be interesting to explore these mechanisms, and the cortical activity associated with the two phases of the mental rotation process by means of, e.g., EEG measurements.

\section{References}

[1] American Psychiatric Association. Diagnostic and statistical manual of mental disorders, 3rd ed., revised. Washington, DC: American Psychiatric Association 1987.

[2] Amorim MA. "What is my avatar seeing?'”: the coordination of "out of body" and "embodied" perspectives for scene recognition across views. Vis Cogn 2003; 10: 157-199.

[3] Amorim MA, Stucchi N. Viewer- and object-centered mental explorations of an imagined environment are not equivalent. Cogn Brain Res 1997; 5: 229-239.

[4] Committeri G, Galati G, Paradis AL, Pizzamiglio L, Berthoz A, LeBihan D. Reference frames for spatial cognition: different brain areas are involved in viewer-, object-, and landmark-centered judgments about object location. J Cogn Neurosci 2004; 16: 1517-1535.

[5] David N, Bewernick BH, Cohen MX, et al. Neural representations of self versus other: visual-spatial perspective taking and agency in a virtual ball-tossing game. J Cogn Neurosci 2006; 18: 898-910.

[6] Easton RD, Sholl MJ. Object-array structure, frames of reference, and retrieval of spatial knowledge. J Exp Psychol Learn 1995; 21: 483-500.

[7] Fossati A, Feeney JA, Donati D, et al. Personality disorders and adult attachment dimensions in a mixed psychiatric sample: a multivariate study. J Nerv Ment Dis 2003; 191: 30-37.

[8] Freeman D. Studying and treating schizophrenia using virtual reality: a new paradigm. Schizophr Bull 2008; 34: 605-610.

[9] Galati G, Pelle G, Berthoz A, Committeri G. Multiple reference frames used by the human brain for spatial perception and memory. Exp Brain Res 2010; 206: 109-120.

[10] Huttenlocher J, Presson CC. Mental rotation and the perspective problem. Cogn Psychol 1973; 4: 277-299.

[11] Kockler H, Scheef L, Tepest R, et al. Visuospatial perspective taking in a dynamic environment: perceiving moving objects from a first-person-perspective induces a disposition to act. Conscious Cogn 2010; 19: 690-701.

[12] Kosslyn SM. Image and brain: the resolution of the imagery debate. Cambridge, MA: MIT Press 1994.

[13] Lambrey S, Amorim MA, Samson S, et al. Distinct visual perspective-taking strategies involve the left and right medial temporal lobe structures differently. Brain 2008; 131: 523-534.

[14] Landgraf S, Krebs MO, Olié JP, et al. Real world referencing and schizophrenia: are we experiencing the same reality? Neuropsychologia 2010; 48: 2922-2930.

[15] Langdon R, Coltheart M. Mentalising, schizotypy and schizophrenia. Cognition 1999; 71: 43-71.

[16] Langdon R, Coltheart M. Visual perspective-taking and schizotypy: evidence for a simulation-based account of mentalizing in normal adults. Cognition 2001; 82: 1-26.

[17] Langdon R, Coltheart M, Ward PB, Catts SV. Visual and cognitive perspective-taking impairments in schizophrenia: a failure of allocentric simulation? Cogn Neuropsychiatry 2001; 6: 241-269.

[18] Oldfield RC. The assessment and analysis of handedness: the Edinburgh inventory. Neuropsychologia 1971; 9: 97-113.

[19] Presson CC. Strategies in spatial reasoning. J Exp Psychol Learn 1982; 8: 243-251.

[20] Raine A. The SPQ: a scale for the assessment of schizotypal personality based on DSM-III-R criteria. Schizophrenia Bull 1991; 17: 555-564.

[21] Riecke BE, Cunningham DW, Bülthoff HH. Spatial updating in virtual reality: the sufficiency of visual information. Psychol Res 2007; 71: 298-313.

[22] Shepard RN, Metzler J. Mental rotation of three-dimensional objects. Science 1971; 171: 701-703.

[23] Sulpizio V, Committeri G, Lambrey S, Zaoui M, Berthoz A, Galati G. Human cortical regions encoding spatial locations in the environment across viewpoint changes. Soc Neurosci 2009; Abstract 380.2/FF100.

[24] Sulpizio V, Committeri G, Lambrey S, Zaoui M, Berthoz A, Galati G. Seeing the world from different views: neural correlates of perspective taking and spatial updating. Soc Neurosci 2010; Abstract 2255.

[25] Vidal M, Lehmann A, Bülthoff HH. A multisensory approach to spatial updating: the case of mental rotations. Exp Brain Res 2009; 197: 59-68.

[26] Vogeley K, Fink GR. Neural correlates of the first-personperspective. Trends Cogn Sci 2003; 7: 38-42.

[27] Vogeley K, May M, Ritzl A, Falkai P, Zilles K, Fink GR. Neural correlates of first-person perspective as one constituent of human self-consciousness. J Cogn Neurosci 2004; 16: $817-827$.

[28] Wan XI, Wang RF, Crowell JA. Spatial updating in superimposed real and virtual environments. Atten Percept Psychophys 2009; 71: 42-51.

[29] Wraga M, Creem SH, Proffitt DR. Updating displays after imagined object and viewer rotations. J Exp Psychol Learn 2000; 26: 151-168.

[30] Zacks JM, Michelon P. Transformations of visuospatial images. Behav Cogn Neurosci Rev 2005; 4: 96-118.

Received February 3, 2011; accepted July 26, 2011 